\title{
Describing color appearance: Hue and saturation scaling
}

\author{
JAMES GORDON \\ Hunter College of City University of New York, New York \\ and Laboratory of Biophysics, Rockefeller University, New York, New York \\ ISRAEL ABRAMOV \\ Brooklyn College of City University of New York, Brooklyn, New York \\ and Laboratory of Biophysics, Rockefeller University, New York, New York \\ and \\ HOOVER CHAN \\ Smith-Kettlewell Eye Research Institute, San Francisco, California
}

\begin{abstract}
Most of the fully elaborated systems for describing color appearance rely on matching to samples from some standard set. Since this is not satisfactory in all situations, various forms of direct linguistic description have been used, ranging from color naming to continuous numerical scaling of sensations. We have developed and extensively applied a particular variant in which subjects use percentage scales to describe their sensations of the four unique hue sensations (red, yellow, green, blue) and of the apparent saturation of colored lights. In this paper we explore the properties of this procedure, including its statistical properties and reliability both between and within subjects, in different contexts. We conclude that the technique is robust, easy to use, and provides direct access to sensory experience.
\end{abstract}

One of the salient features of the world in which we live is that things appear colored, often brightly so. We see a red book on the shelf, rather than a book that, incidentally, appears red. Because color is so rooted in our perceptions, a full description of what we see must include precise statements about it. But, as we have known for three centuries, this cannot be done simply by describing the physical properties of any object, such as the wavelengths of light that it reflects: "For the rays to speak properly are not coloured. In them there is nothing else than a certain power and disposition to stir up a sensation of this or that colour" (Newton, 1704, p. 90).

In standard colorimetry, the color of an object or a light is specified in terms of the additive mixture of three primary lights needed to match it (Wyszecki \& Stiles, 1967). Not only can this be very precise, but it is also conceptually important. Even though the wavelengths of light from the two stimuli-- the sample and the matching mixture-are often drastically different, they elicit identical responses from the visual system and are perceived as being completely equivalent. This severely constrains the mechanisms that can be postulated to account for

This research was supported in part by grants EYO7129 and EYO1428 from the National Institutes of Health, and 661209 from the PSC/CUNY Faculty Research Award Program. Address correspondence to J. Gordon, Department of Psychology, Hunter College of CUNY, 695 Park Avenue, New York, NY 10021 (e-mail: jmghc@ cunyvm. bitnet) color vision. However, colorimetry still describes colors only by means of standardized equivalents and does not describe what they actually look like. For example, the light of a wavelength that appears yellow can be exactly matched by a mixture of two other wavelengths, one that appears red and one that appears green; the "yellow" is specified by the relative intensities of the "red" and "green" in the mixture. Normally, the "yellow" stimulus and the matching mixture both appear identically yellow. But what it looks like also depends on many other factors. If one first stares at a field that appears red and then looks at these stimuli, the single wavelength and the mixture will still look like each other (a color match is unaffected by chromatic adaptation short of considerable pigment bleaching), but neither will look yellowthey will look distinctly greenish.

A variety of color-order systems has been devised to meet the demands for descriptions of color appearance. These systems specify colors "within a limited domain by means of a set of material standards" (reflectance samples or chips) chosen to cover the gamut of colors that one might need to describe (Wyszecki \& Stiles, 1967, p. 475); in this sense, the systems are "objective," but they also include "subjective" elements. In the widely used Munsell system, the scales of hue, chroma, and value have been adjusted so that the chips are uniformly separated along the corresponding sensory dimensions (Newhall, Nickerson, \& Judd, 1943). In use, the particular chip that most closely matches the object 
to be described is found, and the Munsell notation of that chip specifies the object's color. However, unless one is familiar with Munsell notation, it may be necessary to present the actual chip. Even this may not be enough, since the observer's state of adaptation can affect its appearance. Also, the particular mix of wavelengths reaching the eye is the product of the relatively broad reflectance spectra of the pigments that color the chips and the illuminant's spectrum; any change in the illuminant can markedly change a chip's appearance. Ideally, then, the chips must be viewed under a standard illuminant and with a neutrally adapted eye, while the object is viewed under the particular conditions for which one wants to specify its appearance. All of this can become very cumbersome, since it may require looking back and forth between them and even using separate eyes for the chips and the object.
We opt for a more direct approach - we ask the observer what the object looks like. However, the way in which the observer is asked to describe a color must be carefully chosen.

The terms used for a single hue, such as pea green, sea green, olive green, grass green, sage green, evergreen, invisible green, are not to be trusted in ordering a piece of cloth. They invite mistakes and disappointment. Not only are they inaccurate: they are inappropriate. Can we imagine musical tones called lark, canary, cockatoo, crow, cat, dog, or mouse, because they bear some distant resemblance to the cries of those animals? (Munsell, 1905, p. 8)

To meet such objections, we have been developing a controlled percentage-estimation procedure in which observers numerically rate their color sensations. For simplicity, we ignore the surface properties of colored
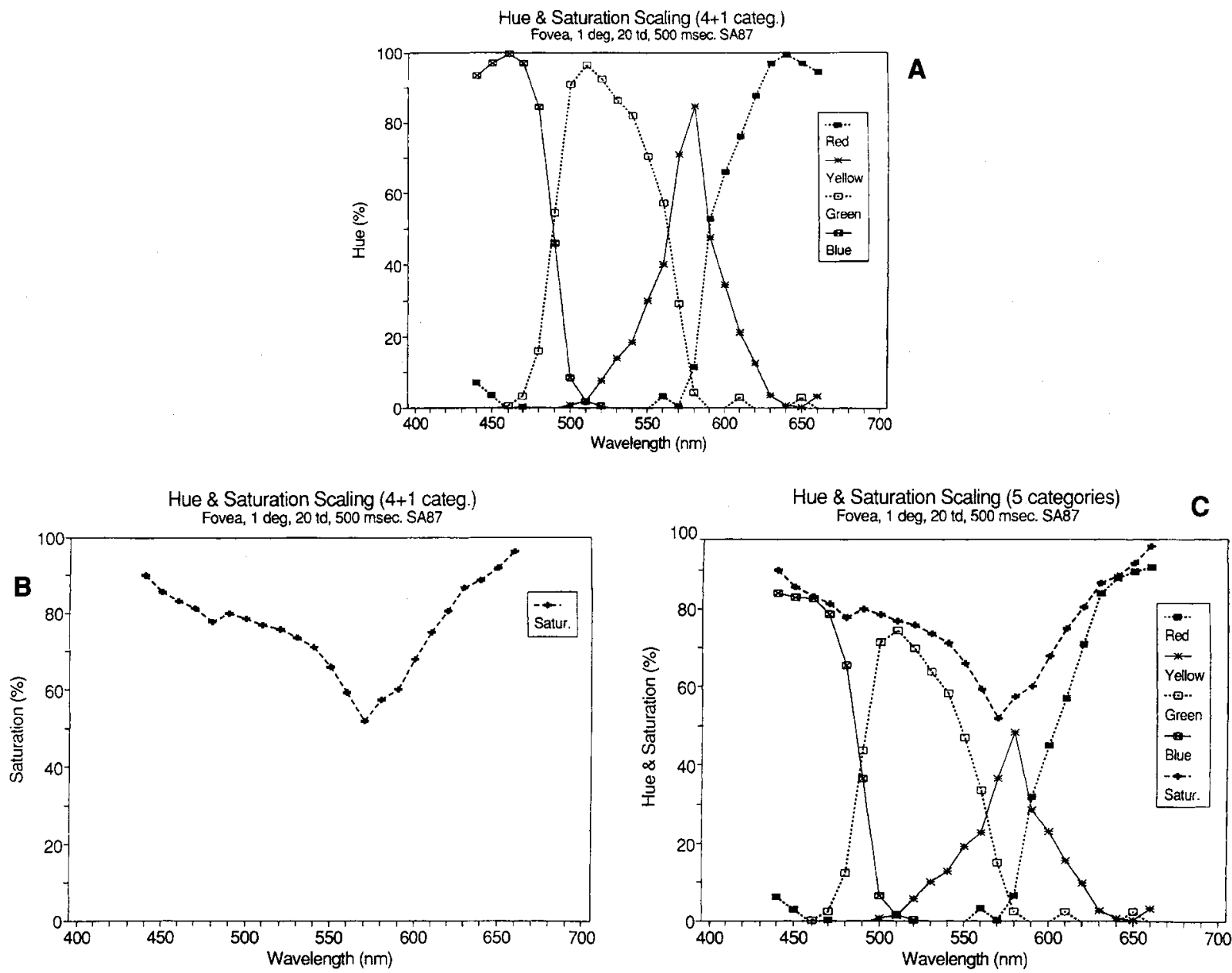

Figure 1. Hue and saturation functions from a representative subject. Stimuli were foveally viewed flashes of monochromatic light, equated for a low level of photopic intensity and seen against a dark background. After each flash, a subject rated percentages of the four unique hues (red, yellow, green, blue) in the sensation; these had to sum to $100 \%$, or to zero if there was no hue. The subject also stated, as a separate number, the apparent saturation (i.e., percentage of the entire sensation that was chromatic). We term this method " $4+1$ categories." $(A) M e a n(N=32)$ hue functions. (B) Mean saturation function. (C) Curves from A and B replotted in "5-category" format. At each wavelength, the hue values in $A$ have been multiplied by the pencent saturation experienced at that wavelength; thus, the rescaled hue values sum to the saturation value at each wavelength. (Before averaging, an aresine transform was applied to the raw data from each trial in order to equalize variances; see text for details.) 
Table 1

Effects of Arcsine Transform on Means and Variances of Hue and Saturation Scaling Responses and Group Means of Individual Subjects' Means and Variances Before and After Transform

\begin{tabular}{|c|c|c|c|c|c|c|}
\hline & Red & Yellow & Green & Blue & Saturation & Row Mean \\
\hline \multicolumn{7}{|c|}{ Means } \\
\hline Mean means (untrans) & 27.1 & 18.2 & 30.2 & 24.5 & 80.1 & 36.0 \\
\hline Mean means (trans) & 26.3 & 21.1 & 28.6 & 23.9 & 72.8 & 34.6 \\
\hline Untrans/trans & 1.0 & 0.9 & 1.0 & 1.0 & 1.1 & 1.0 \\
\hline \multicolumn{7}{|c|}{ Variances } \\
\hline Mean variance (untrans) & 56.4 & 100.9 & 144.1 & 71.4 & 93.6 & 93.3 \\
\hline Mean variance (trans) & 49.8 & 83.4 & 110.7 & 58.3 & 57.2 & 71.9 \\
\hline Untrans/trans & 1.1 & 1.2 & 1.3 & 1.2 & 1.6 & 1.3 \\
\hline Variance of variance (untrans) & $14,529.1$ & $34,022.0$ & $67,256.3$ & $43,228.1$ & $10,765.3$ & $33,960.1$ \\
\hline Variance of variance (trans) & $9,035.4$ & $15,837.3$ & $28,907.6$ & $17,941.7$ & $3,048.2$ & $14,954.0$ \\
\hline Untrans/trans & 1.6 & 2.1 & 2.3 & 2.4 & 3.5 & 2.4 \\
\hline \multicolumn{7}{|c|}{$\begin{array}{l}\text { Note-Group data from } 6 \text { subjects. Each value is the mean of the corresponding values from the individual subjects. Hues and } \\
\text { saturations of each of } 23 \text { stimuli were scaled a total of } 32 \text { times by each subject, yielding } 115 \text { ( } 5 \text { categories } \times 23 \text { wavelengths) } \\
\text { individual means and variances. The values were computed from trial-by-trial responses before and after the arcsine transfor- } \\
\text { mation. Values were collapsed together within a response category. Stimuli were monochromatic lights, } 440-660 \text { nm, foveal, } \\
1^{\circ}, 500 \mathrm{msec}, 20 \mathrm{Td} \text {. }\end{array}$} \\
\hline
\end{tabular}

stimuli and limit ourselves to "aperture colors" or "light mode of perception" (Evans, 1964). In this case, it is generally accepted that sensations vary along three approximately independent dimensions: hue, saturation, and brightness (Boynton, 1979; Hunt, 1975; Hurvich, 1981); as in many studies of color vision, we hold brightness approximately constant and study how hue and saturation are related to changes in the physical characteristics of our stimuli. We do this with a specific form of numerical estimation. There is, of course, a considerable history of using estimation techniques to obtain immediate and direct measures of sensation (e.g., Marks, 1974). However, the details of the method that subjects use to assign numbers to sensations must be considered carefully (e.g., Gescheider, 1988; Gescheider \& Bolanowski, 1991; Poulton, 1979).

The generic technique of hue and saturation scaling that we use was first described by Jameson and Hurvich (1959) and has since been used to study many aspects of color vision: the effect of size and retinal locus of stimulation (Abramov, Gordon, \& Chan, 1991, 1992; Boynton, Schafer, \& Neun, 1964; Gordon \& Abramov, 1977; Kaiser, 1968), color appearance following chromatic adaptation (Jacobs \& Gaylord, 1967; Sobagaki, Yamanaka, Takahama, \& Nayatani, 1974), color anomalies (Pokorny \& Smith, 1977; Smith, Pokorny, \& Swartley, 1973), duration of stimulation (Luria, 1967), and the change in hue with intensity (Boynton \& Gordon, 1965). Also, The Natural Color System, adopted in 1979 by the Swedish Standards Institute, is the only "appearance" or color-order system whose reflectance chips were chosen directly from hue and saturation scaling of many stimuli (Hard \& Sivik, 1981).

Despite wide use, there is no single system of hue and saturation scaling - everyone devises his or her own variant. Because seemingly minor variations in procedure, such as changing stimulus range, can grossly change the psychophysical relation, we ask: To what extent are hue and saturation scaling subject to these problems? There is no detailed analysis of the techniques. We present here our tests of the method we use. In some cases we have done experiments that were designed to test specific properties. In other cases we have reanalyzed data from other studies, whose primary goals were not to test the methods as such. We will show that hue and saturation scaling, at least as we use it, is a very robust procedure; it is reliable and easy to use, and is relatively free of many of the biases associated with some forms of estimation procedures.

\section{Necessary and Sufficient Categories}

There are many terms for the different hues; the list depends on the particular language. However, unlike most other linguistic categories, hue terms seem to have the same denotations, as defined by "best exemplar," across all languages that have the lexical equivalents (Berlin \& Kay, 1969; Heider, 1972; Kay \& McDaniel, 1978). This has led some to conclude that the basic hue terms closely reflect the responses of sensory areas of the nervous system that code color sensations (Ratliff, 1976). But what is the set that is necessary and sufficient for a complete description of all hues? Until quite recently, the answer depended on theoretical orientation. Beare (1963), following Graham (1958), used six terms, whereas Jameson and Hurvich (1959), in the tradition of Hering (1920), argued in favor of two opponent hue pairs: red-green and yellow-blue. Today it is generally accepted that hues (at least of aperture colors) can be described completely by using only the four terms that Hering postulated as corresponding to the phenomenologically unique hue sensations: red $(R)$, yellow $(Y)$, green (G), and blue (B).

A clear demonstration that the above four terms are both sufficient and necessary descriptors was given by Sternheim and Boynton (1966). They began with the four categories R, Y, G, B, plus orange, and asked sub- 

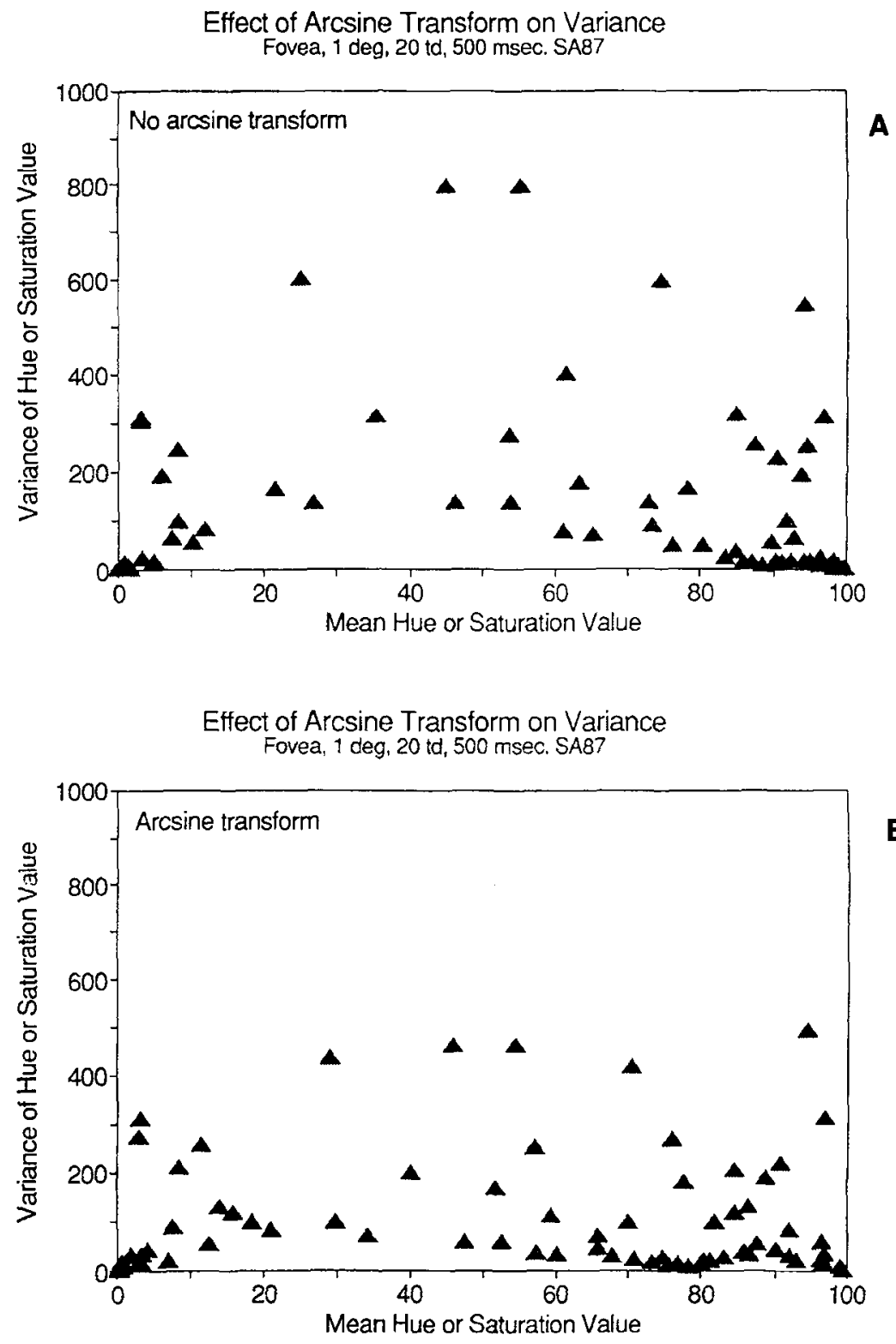

Figure 2. Use of an arcsine transform of each scaling datum (hue or saturation value on each trial) in order to equalize variances. (A) Variances associated with each mean from the data set shown in Figures $1 \mathrm{~A}$ and $1 \mathrm{~B}$ plotted against the means ( 32 repeats per mean); the statistics are for the raw, untransformed scaling responses. (B) The same data set, except that each response was transformed by an arcsine correction before averaging or calculation of variances; in fact, these are the means plotted in Figure 1.

jects to describe the percentage of their hue sensations in each of the five categories in response to monochromatic lights. On different sessions, one or more of the terms was eliminated from the set of permissible categories, but the responses from the remaining categories no longer had to sum to $100 \%$. The rationale was that when an unnecessary category was eliminated, sensations would be apportioned readily among the other categories, with the total for any stimulus still adding up to 100. However, when a necessary term was eliminated, the proportion of sensation that it usually would have denoted could not be included in the remaining categories; the missing color function could be computed from the rest of the data. Of their five initial terms, only orange was unnecessary. Similarly, purple and gray are not necessary (Fuld, Wooten, \& Whalen, 1981).

A complete description of an aperture color also needs a term for the achromatic component of the sensation; if the percentage of the sensation represented by that term is subtracted from the total of $100 \%$, it can be 

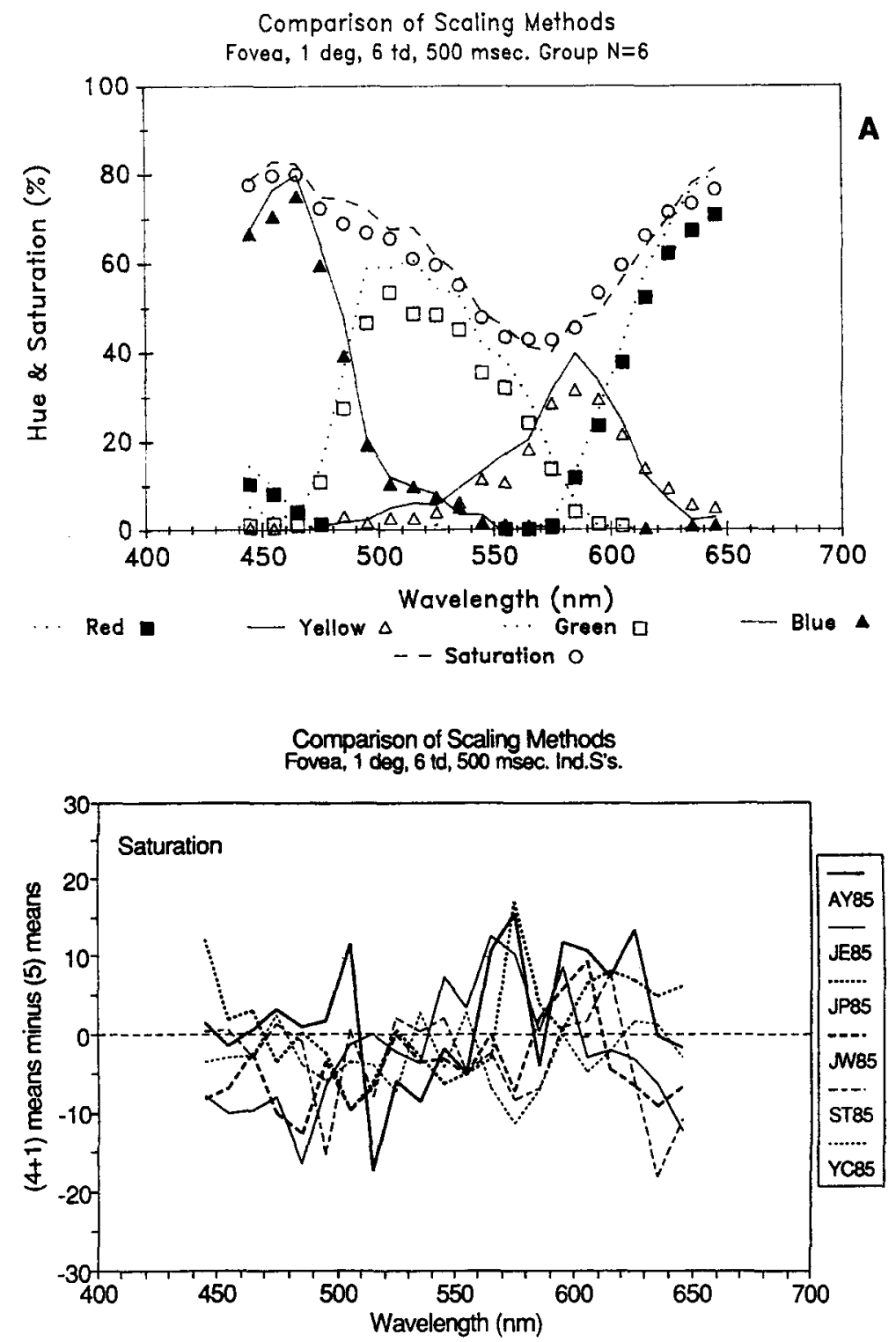

Figure 3. Comparison of " $4+1$ " and "5-category" scaling methods; in $4+1$, hues and saturation are described separately, but in the 5-category method the percentages of the hues together with the achromatic sensory component must jointly sum to 100. The same 6 subjects alternated between the two techniques in a counterbalanced design. (A) Symbols are the group means using $4+1$ categories, but rescaled by saturation, as in Figure 1C. The curves are means using the 5-category method; the achromatic values were subtracted from 100 to obtain saturation. (B) Individual difierence functions for saturation obtained from the two methods. (An arcsine transform was applied to the raw data from each trial in order to equalize variances; see text for details.)

called apparent saturation. This is not related exclusively to stimulus purity-even spectral lights of arbitrarily high purity can vary in apparent saturation. When the stimulus is seen in isolation, with a dark surround, the achromatic or desaturating component of the sensation can be called white, and when the surround is brighter than the stimulus, it is black. Incidentally, it had been thought that when a stimulus that appeared yellow was surrounded by a brighter achromatic area, brown had to be added as a necessary hue category (Fuld, Werner, \& Wooten, 1983). But it is now clear that even under these conditions the only essential terms are R, Y, G, B, and achromatic (Quinn, Rosano, \& Wooten, 1988).

Our conceptual framework for scaling these sensory quantities can be broadly described as follows (Abramov \& Gordon, 1994). There are separate chromatic and achromatic pathways that process inputs from the three spectrally distinct cone types in the retina. The chro- 
matic mechanisms must somehow preserve differences in relative responses of different cone types in order to preserve any information about stimulus wavelength; this is because each of the different cone types can only signal the rate at which it absorbs photons. The nervous system carries out this comparison by means of spectrally opponent neurons that are stimulated by one type of cone and inhibited by another. The chromatic pathway is divided into separate $\mathrm{R}, \mathrm{Y}, \mathrm{G}$, and $\mathrm{B}$ mechanisms; these are the unique hue sensations, each of which is a unitary category and cannot be further divided. Achromatic pathways are represented by spectrally nonopponent systems that simply combine cone responses. The ratio of chromatic to total responses (i.e., chromatic plus achromatic) represents the degree to which a stimulus appears saturated. But these mechanisms are not neces- sarily the same as the opponent and nonopponent cells, or parvo- and magno-cellular units that are currently known, even though these neurons must provide the inputs to the sensory pathways with which we are concerned (e.g., De Valois, Abramov, \& Jacobs, 1966; Ratliff, 1976; Zrenner et al., 1990).

Our linking hypothesis (Teller, 1990) is that the four chromatic processes are the channels for the four unique hue sensations; each hue sensation is directly related to the pattern of responses across these cells. They constitute internal "standards" for the hues, and a unique sensation occurs when only one of them responds to a stimulus. Thus, a response of "red" reflects responses of some unitary mechanism, even though that mechanism may itself receive inputs from many submechanisms. For example, short- and long-wavelength redness may
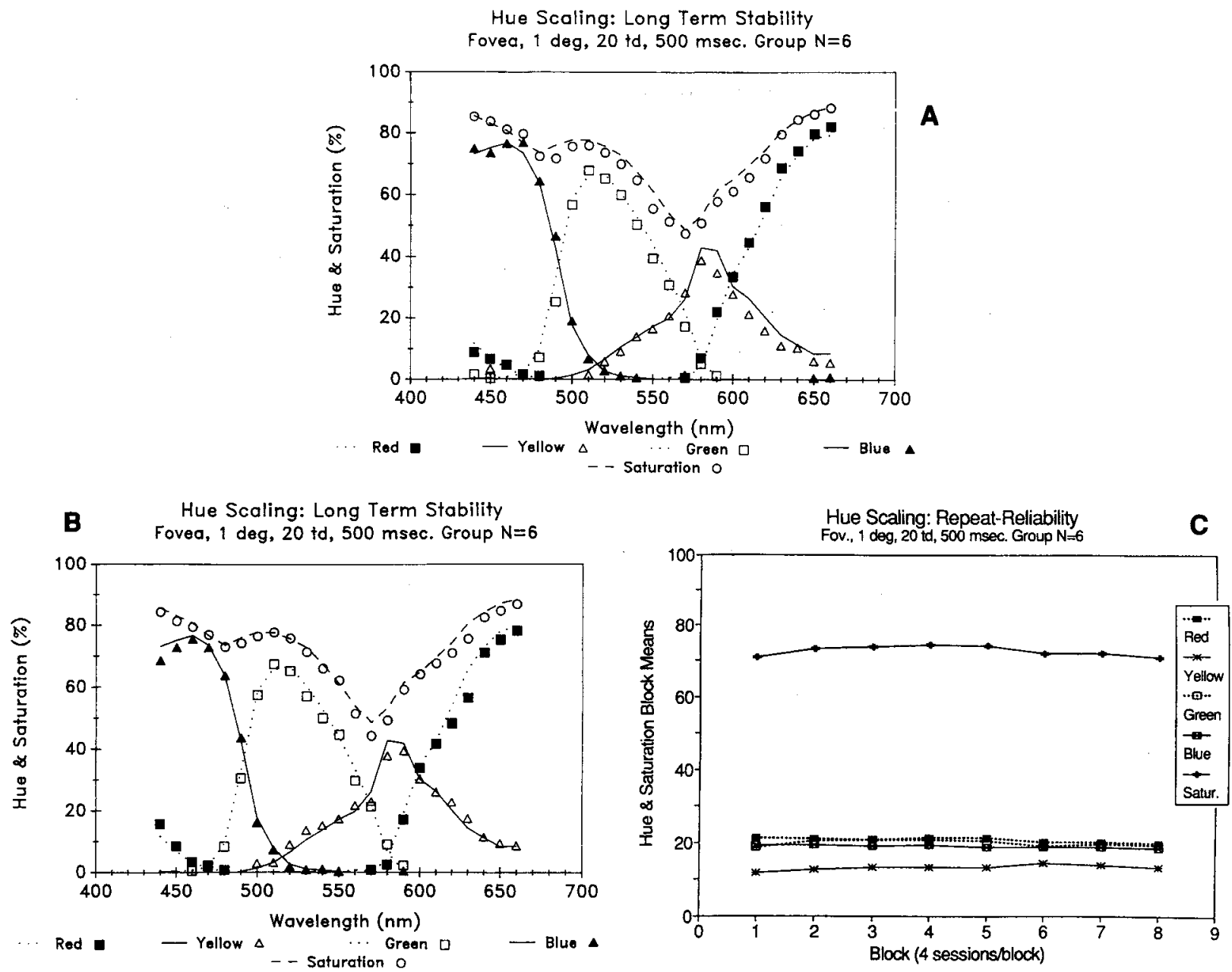

Figure 4. Long-term stability of hue and saturation values. Group data $(N=6)$ from subjects who scaled foveal and peripheral stimuli across 32 sessions spaced over several months. The figure shows results only for the foveal stimuli, each of which appeared once in each session. (A) Symbols are the group means for the first four sessions; curves are the means from all 32 sessions. (B) Symbols are the group means for the last four sessions; curves are the means from all 32 sessions. (C) Group means of the responses in each sensory category in successive blocks of four sessions; that is, for each point, all the responses in a category (e.g., red) were averaged for each block. (An aresine transform was applied to the raw data from each trial in order to equalize variances; see text for details.) 

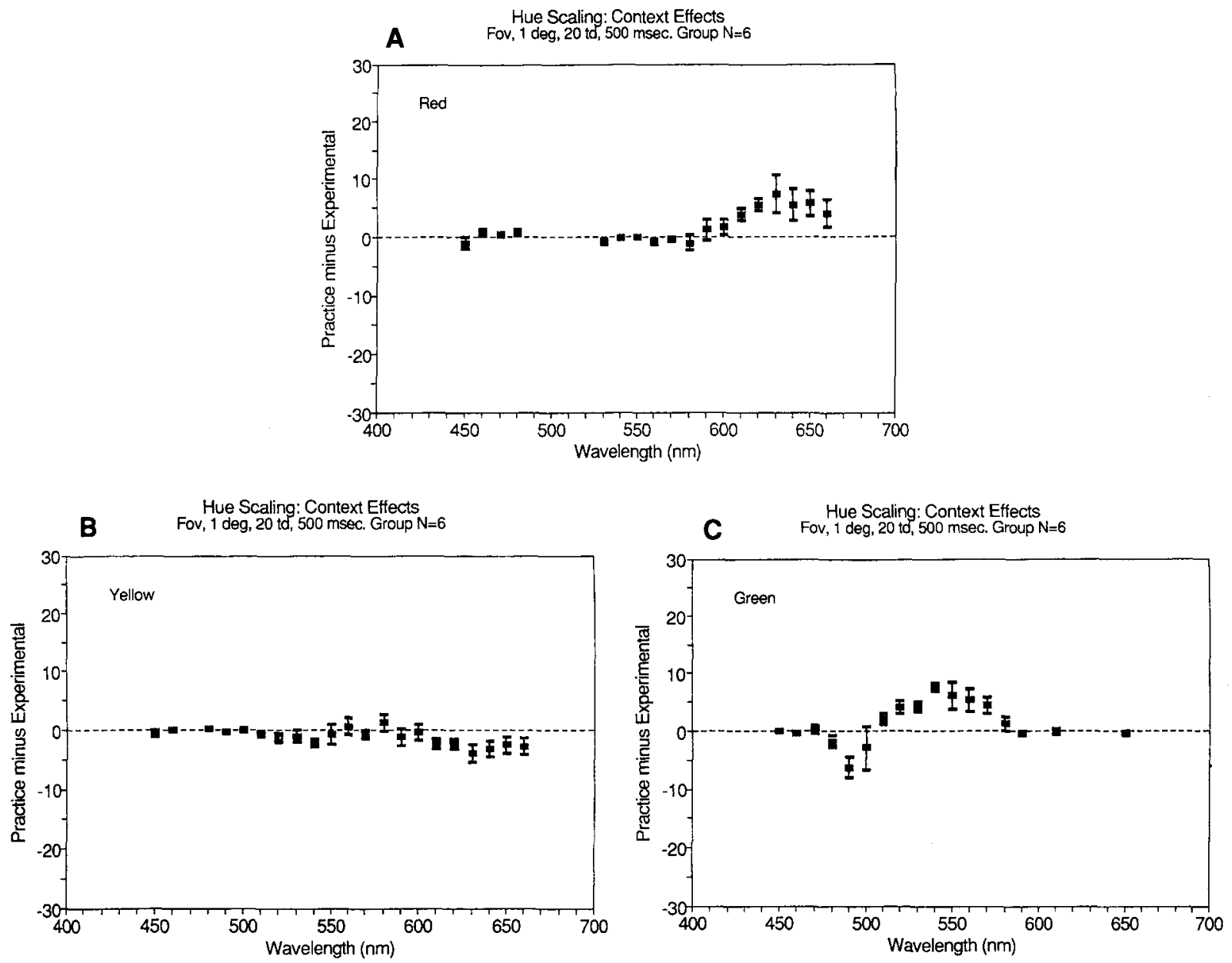

Hue Scaling: Context Effects
Dov, 1 deg, 20 do, 500 msec. Group $\mathrm{N}=6$
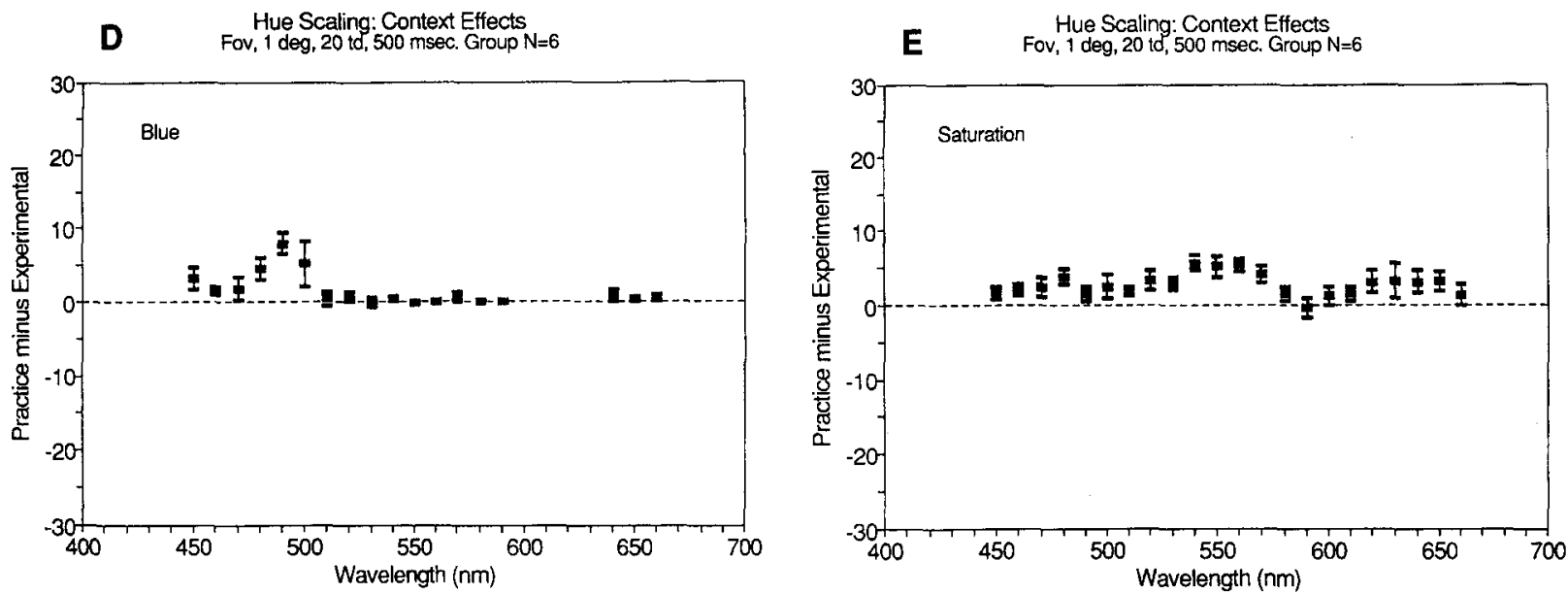

Figure 5. Effects of context on hue and saturation values. Data are from the same study used for Figure 4. Each session began with a series of "practice" stimuli presented one after the other and only in the fovea; "experimental" trials included the same stimuli as in "practice," but they were embedded in a sequence of other stimuli seen at various peripheral loci. (A-E) Differences between practice and experimental trials for each subject were averaged in each sensory category; error bars represent \pm 1 SEM of the group means. (An arcsine transform was applied to the raw data from each trial in order to equalize variances; see text for details.) 


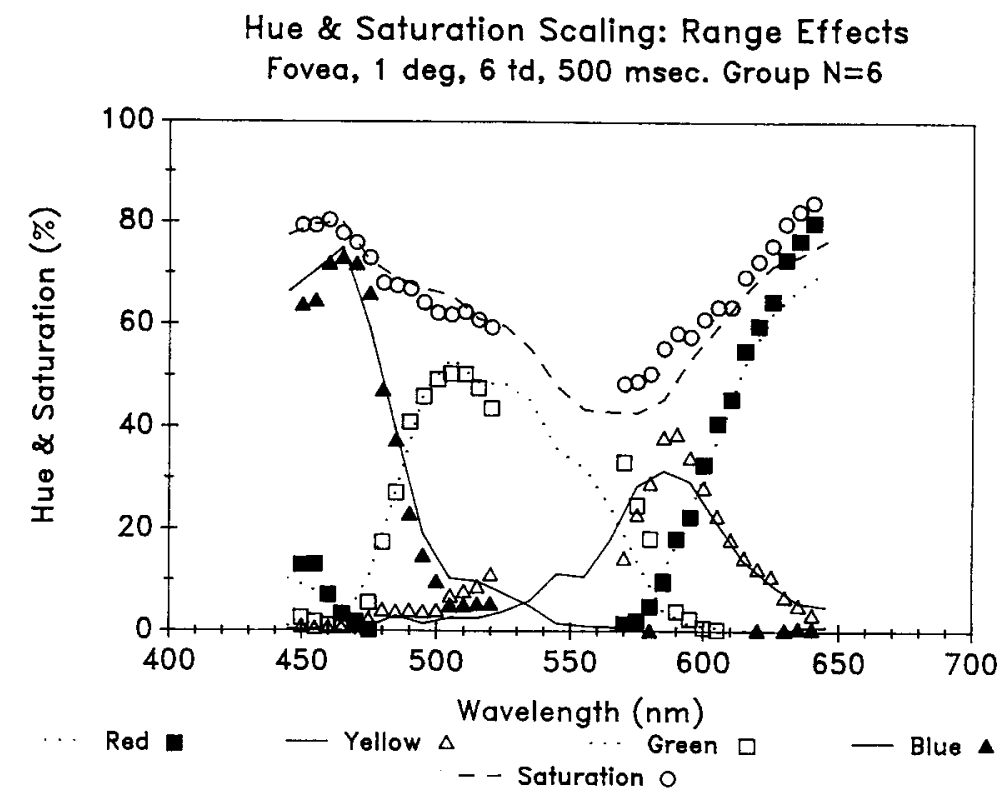

Figure 6. Effects of stimulus range on hue and saturation values and group means from subjects who viewed foveal stimuli. In some sessions the stimuli were evenly divided across the spectrum; in others they were taken either from the shorter or longer wavelength portions. In restricted-range sessions, stimuli were spaced closer in wavelength so as to keep the number of trials per session approximately constant. Symbols are the means from restricted-range sessions, and curves are the means from full-spectrum sessions. (An ancsine transform was applied to the raw data from each trial in order to equalize variances; see text for details.)

be due to different processes, but ultimately they must come together in a final common pathway, because the final appearance is the same-red. The four unique hue terms can be used to examine the properties of these mechanisms. Subjects do not need special training to use these terms since they refer to internal standards, as evidenced by the fact that the denotations of these terms are universal. Apparent saturation is based on the relative strengths of the responses by the chromatic and achromatic processes.

\section{SCALING PROCEDURE}

For all the studies considered here, subjects scaled the appearance of small uniform fields of monochromatic light presented as brief flashes against a dark background; all lights were equated for retinal illuminance, and the time between flashes was $15-20 \mathrm{sec}$ to minimize carryover of sensory effects. (Details are given with each set of results.) Different groups of subjects were used, although some, as will be noted, took part in more than one study. The age range for all the subjects was 16 to 49 years. All were screened with the Dvorine PseudoIsochromatic Plates, the Farnsworth Dichotomous Test for Color Blindness, Panel D-15, and the FarnsworthMunsell 100 Hue Test. Unless otherwise stated, all the subjects knew the aim of the study in which they participated as subjects and experimenters.
We call our basic method " $4+1$ categories." After each stimulus, the subject reports the percentages of his/her sensation of $\mathrm{R}, \mathrm{Y}, \mathrm{G}$, or B for a total of $100 \%$; the subject then states apparent saturation (S), or chromatic content, as a percentage of the sensation elicited by the stimulus that was just seen. Typical instructions (there are minor variations for each study or optical system), which are read before each session regardless of the subject's expertise, are:

A beep will warn you to look directly at the center of the space surrounded by the four fixation dots. After each beep, a flash of light will be shown to you. After each flash, describe the sensation produced in you by the light. First, divide your sensation into two parts. The first part consists of hue. You must divide your sensation of hue into red, yellow, green, or blue. These are the only words you may use to describe the hue. If you wish, you may use pairs of names to describe a hue. When you are describing your sensations of hue, please state them in percentages. The percentages you assign to these words must add up to 100 . For instance, your sensation might be $40 \%$ red and $60 \%$ yellow, or $86 \%$ green and $14 \%$ yellow, or $95 \%$ blue and $5 \%$ red, and so on. It can also be $100 \%$ of one of the hues.

Think carefully about your answer and try to be as precise as possible. Remember that the term hue refers to your own sensation elicited by the light. You are not being asked how you might create the particular hue you saw. You are being asked to describe your sensation. Please 
A Hue Scaling: Naive vs Experienced Subjects

Fovea, 1 deg, $20 \mathrm{td}, 500$ msec. Group $N=6$

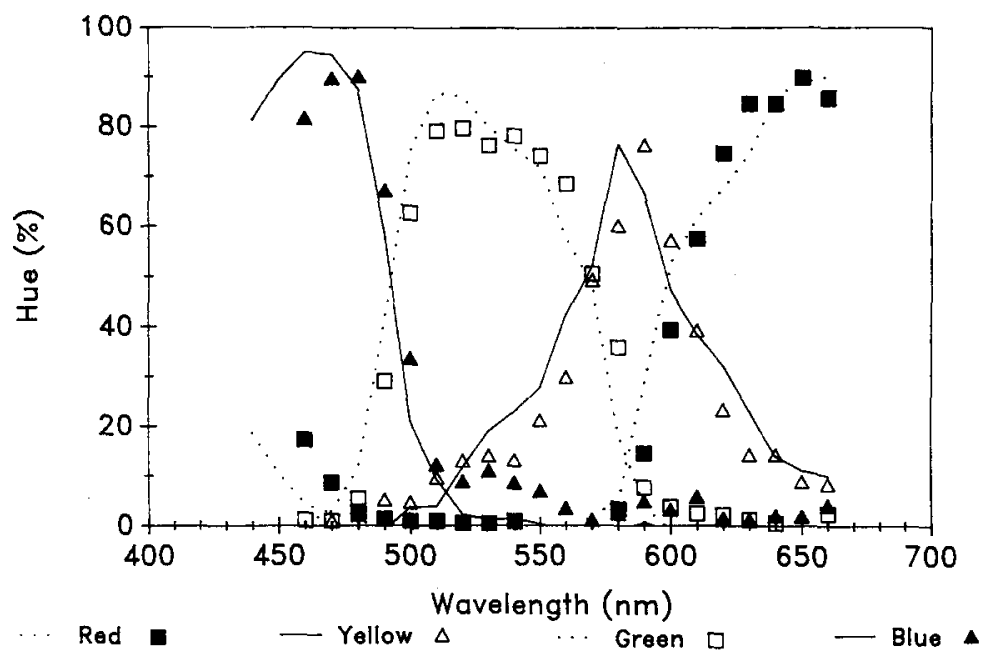

B Saturation Scaling: Naive vs Experienced Subjects

Fovea, 1 deg, 20 td, 500 msec. Group $N=6$

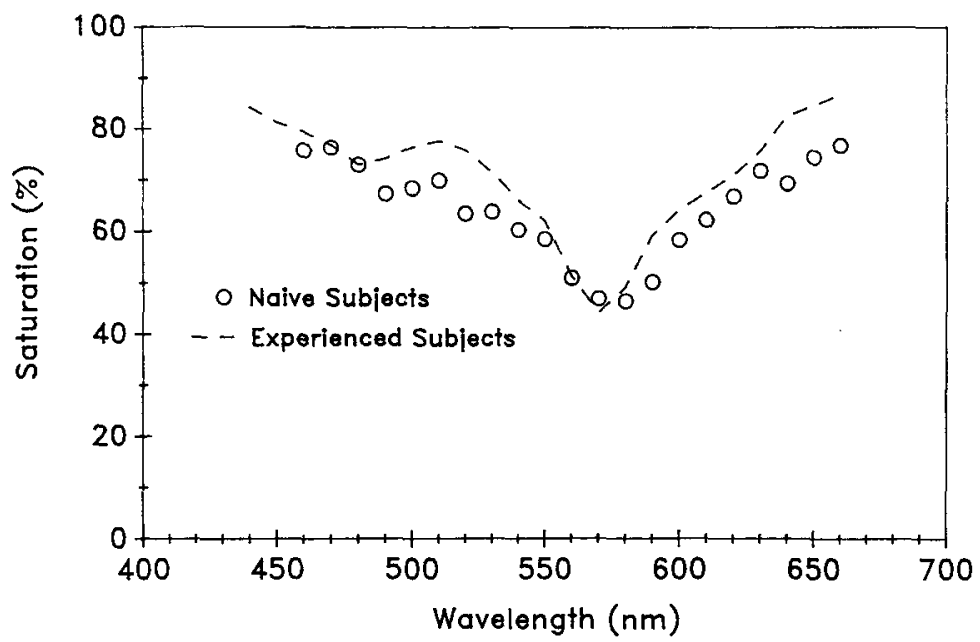

Figure 7. Mean hue and saturation scaling functions from groups of experienced and experimentally naive subjects viewing foveal stimuli; symbols are from the naive subjects, curves are from experienced observers. (A) Hue functions (4 + 1 category format). (B) Saturation functions. (An aresine transform was applied to the raw data from each trial in order to equalize variances; see text for details.)

note that there are no right or wrong answers. You are simply describing your sensation.

The second part of your sensation is not hue; it is achromatic. After describing the hue of your sensation, you must consider what percentage it formed of your entire sensation; that is, what is the percentage of chromatic versus achromatic-plus-chromatic sensations? This value is apparent saturation. It refers to the strength or concentration of hue in your total sensation. A total absence of hue would be represented by $0 \%$ saturation and a total absence of any achromatic sensation would be $100 \%$ saturation.
The first few trials will be practice trials. They are done to familiarize you with the procedure. We will tell you when they end and the experimental trials begin. The session will last about 1 hour. Is everything clear?

In response to questions, subjects are encouraged to treat each category as a continuous scale and not limit themselves to just a few values. No specific training is given other than simple practice without any feedback, nor are any of the hue terms defined beyond their common, everyday meanings. No named examples are ever 
shown as "standards"- that would simply tell us how well subjects remembered our definitions rather than what they saw. The most problematic concept for subjects is "saturation." Beyond the descriptions in the instructions, we might offer: "Think of your total sensation after a flash as something contained in a bucket. Now pour a little bit of a hue into it and stir. What has happened to the saturation? Now add a little bit more and stir. Again, how has saturation changed?"

The goal was to find a method that would be easy for subjects while still preserving the relations among the different sensory qualities, since the color sensation is a cross-category comparison. Many of the problems with magnitude scaling are dealt with in the context of a sensory dimension that varies along some intensive continuum (e.g., brightness, loudness; see Gescheider, 1988). Ours differs in that it deals first with subjects' decisions about "which" category to use, but sensory magnitude can vary within a category (e.g., amount of red sensation). Subjects express their judgments using percentages, because they are comfortable with them and use them all the time outside the laboratory. Note that sub- jects are not given any modulus to anchor their judgments. Also, subjects are not told that within a category they must assign numbers to reflect sensory ratios; the only requirement, which is implicit, is that they take whatever numbers they would have assigned to sensations in each category and then tell us the ratios of these values between categories. This may make the demands on the subjects more like those of the absolute magnitudeestimation techniques that have been advocated (e.g., Gescheider \& Hughson, 1991; Zwislocki, 1983; Zwislocki \& Goodman, 1980).

Figure 1 shows data from a typical subject from a lengthy study of color vision across the retina (Abramov et al., 1991). In each session, stimuli of different sizes and locations were randomly intermixed, but on each of the 32 sessions a particular set of stimuli was presented as part of the random stimulus sequence: foveally presented flashes of monochromatic lights, ranging from 440 to $660 \mathrm{~nm}$ in $10-\mathrm{nm}$ steps. All these stimuli subtended $1^{\circ}$ of visual angle, were equated for a retinal illuminance of $20 \mathrm{Td}$, and were seen against a dark background. (In this and all subsequent figures, an arcsine
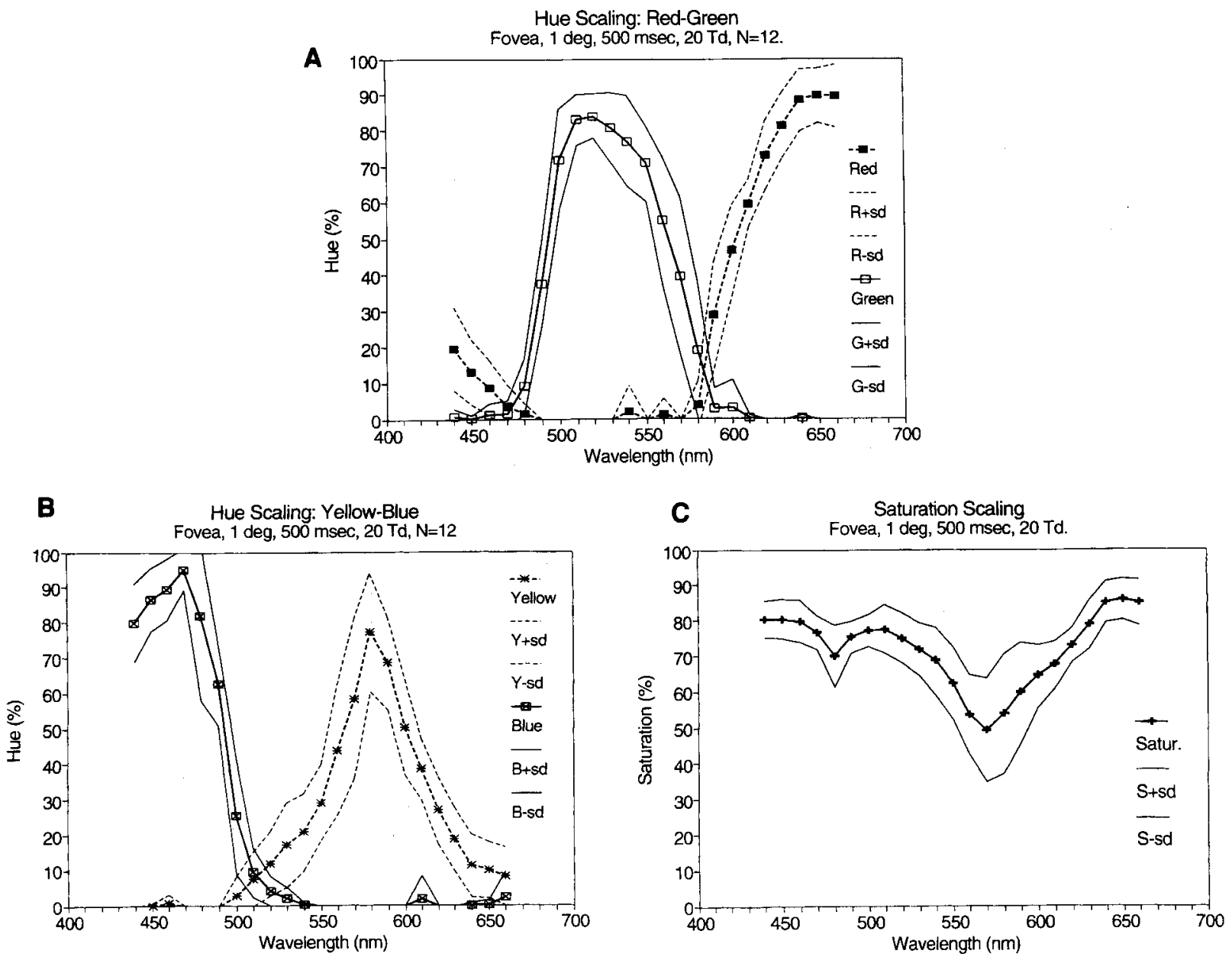

Figure 8. Population variation in hue and saturation scaling. Group means, shown in $4+1$ category format, from 12 subjects; error zones represent \pm 1 estimated standard deviation of the population: (A) red and green, (B) yellow and blue, (C) saturation. (An aresine transform was applied to the raw data from each trial in order to equalize variances; see text for details.) 
Hue \& Saturation Scaling: Monolingual vs Bilingual Subjects

Fovea, $1 \mathrm{deg}, 20 \mathrm{td}, 500$ msec. Group $\mathrm{N}=6$

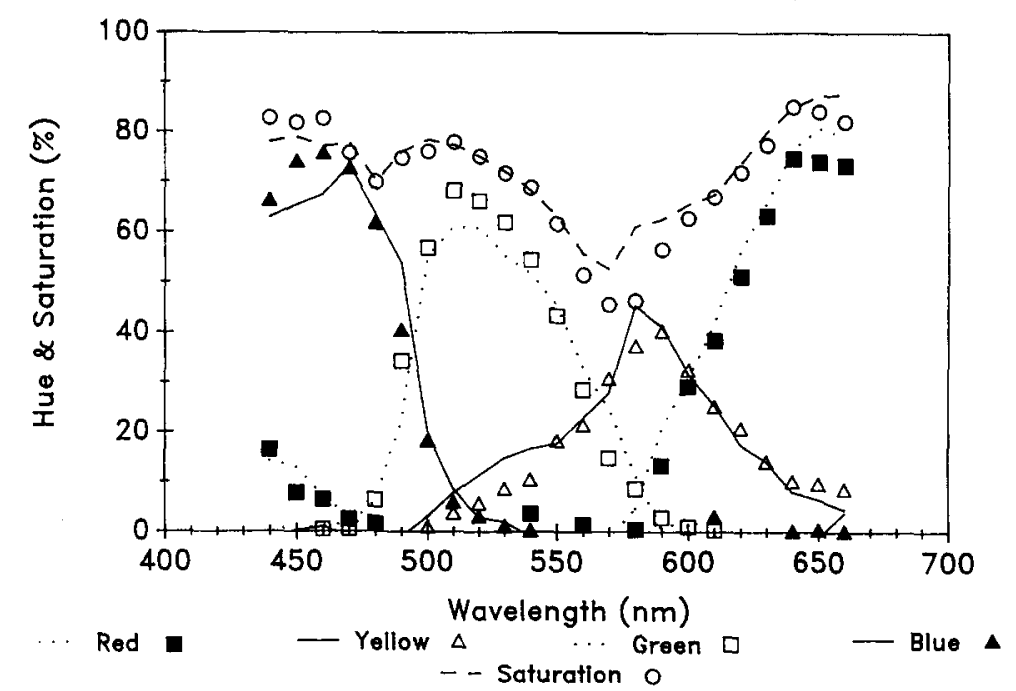

Figure 9. Effects of language background on hue and saturation scaling. Symbols are the means of a group of bilingual subjects (for whom English was the second language) who used English in the experiment; curves are for a group of monolingual English speakers. (An aresine transform was applied to the raw data from each trial in order to equalize variances; see text for details.)

transform was applied to all the raw data. The nature of this transform and the reasons for using it are given in the section on percentage scales.) In Figure 1A we see, starting from the longer wavelengths, that the hues elicited by the stimuli were first mostly $\mathrm{R}$, shading to $\mathrm{Y}$, and in turn shading to $G$, then $B$, and finally back to some $R$ at the shortest wavelengths. Note that $R$ and $G$ are largely mutually exclusive, so that when neither is present in the sensation we have either a wavelength that appears uniquely $\mathrm{Y}$ or another wavelength that appears uniquely B. Similarly, B and Y are opposites and they are absent at unique $G$. The slight overlap of $R$ and $G$ in the figure is due entirely to intertrial variability. We have not encountered a reliable subject who has used both $\mathbf{R}$ and $\mathrm{G}$ for any one trial. Occasionally, however, some subjects (not the one in this figure) use $Y$ and $B$ together, but only together with $G$ as the major response (similarly, see Boynton \& Gordon, 1965; Gordon \& Abramov, 1977). On debriefing, all our subjects who used these three terms said that the stimulus appeared "mottled," with some parts $G$ and B and others $G$ and $Y$. In our earlier work there were no "forbidden" combinations of hue terms. However, because of the extreme rarity of the pairing of $R$ with $G$ and $Y$ with $B$, we now sometimes add the following to the first paragraph of the instructions given previously: "The only limitation is that you may not pair red with green, or pair yellow with blue; all other combinations are allowed." Figure 1B shows the spectral variation in saturation. Note that although all lights were equally pure and were narrow band, saturation still varied; hence, we should discuss "apparent sat- uration" rather than the quantity that varies strictly with purity.

There is a problem with the presentation in Figure 1. For example, $600 \mathrm{~nm}$ appears $66 \% \mathrm{R}$ and $34 \% \mathrm{Y}$. But that stimulus also appears somewhat desaturated-it is only $67 \% \mathrm{~S}$. This possibly misleading separation of saturation and hue is even more glaring in conditions in which everything is very desaturated. To combine both aspects of the sensation, we rescale the hues according to the associated saturation values so that the sum of the hues equals saturation; this is shown in Figure 1C, where $600 \mathrm{~nm}$ elicits a sensation that is $44 \mathrm{R}$ and $23 \mathrm{Y}$. Before dealing with the legitimacy of this rescaling, however, we must consider the particular form of the numerical scales that we use.

\section{Percentage Scales}

We now deal with a relatively minor and well-known problem: percentage scales are bounded. And with such scales, the variances could well vary with the mean, which is least at the extremes. Thus, the means of sensations that are predominantly of one hue will seem more reliable than those of intermediate hues because their variances are typically smaller. We argue that this is an artifact imposed by using bounded scales. But in order to compare sensations elicited by different stimuli, or to compare the effects of different experimental manipulations, it is desirable to have sensory scale values that are equally reliable, regardless of the particular hue ratio. Moreover, one of our goals is to use hue scaling to generate a color space with a uniform metric so that 
equal distances in any direction represent equal perceptual changes. For this, we clearly require values with equal variances.

To investigate the uniformity of variance in hue scaling data, we used the raw data that were transformed to produce Figure 1. For each stimulus, we averaged the trial-by-trial hue and saturation scaling responses; in Figure $2 \mathrm{~A}$ we plot the variances associated with each of these means, regardless of category. If all the means were equally reliable, we would expect the distribution in Figure $2 \mathrm{~A}$ to be rectangular, which it clearly is notvariances are indeed lower for means close to 0 or $100 \%$, many of which lie over each other and are obscured.

A method for normalizing variances of proportions is to apply an arcsine transform to each datum before carrying out any other manipulations, such as averaging (Winer, 1971). The specific form that we used was: Sensation $\%=((2 \times$ arcsine (square root(untransformed sensation $\% / 100)$ )) $/ \mathrm{pi}) \times 100$. The limits of the scale values transformed in this way are still 0 and 100 . Since our percentages are not derived from binomial processes, it is an empirical question whether this transform is useful. Figure 2B shows the effects of this transform; overall, variances are reduced and the distribution is more nearly rectangular.

The study from which the data of Figure 2 are derived had 6 subjects. We repeated the above procedures for each subject. The transform we use should not distort mean scaling functions, such as those in Figure 1. To test this, we averaged together all the mean responses in each hue category and saturation before and after transforming the responses from individual trials. A summary of the results is given in Table 1; this includes only group data because none of the subjects showed a different pattern. The ratios of these untransformed mean category responses to the means from transformed values are approximately 1 in all cases, implying no distortion of the mean functions. Note that this is not simply because the mean responses in each category were symmetrically distributed above and below $50 \%$. For example, all saturation means were above 50 and most yellow means were below 50, and yet in both cases the ratios of untransformed/transformed means were very close to 1 . We also averaged all the variances in a category before and after transformations, and, in this case, mean variance after transform is always less. Also, the variance of the set of transformed variances is reduced, demonstrating that the distribution of variances is made more uniform by the transform. On the basis of this, we apply the arcsine transform to all our data prior to any further analysis; for example, the data in Figures $1 \mathrm{~A}$ and $1 \mathrm{~B}$ were so transformed before reapportioning the data according to their saturation values (Figure 1C).

\section{4+1 Versus 5-Category Procedures}

At one time we had used a 5 -category procedure in which subjects directly produced data, as in Figure 1C (Gordon \& Abramov, 1977). The categories were R, Y,
G, B, and achromatic, all of which had to sum to $100 \%$ for each stimulus; saturation was calculated by subtracting the achromatic value from 100 . We changed to $4+1$ categories because the subjects found it easier. But do the two procedures yield comparable results? Can we convert data from one procedure into the other's format, as was done in Figure 1? Such conversion assumes that subjects compare sensations to their internal standards (the separate hue mechanisms) and can specify ratios of activity among them. For instance, the ratio of $R / Y$ at $600 \mathrm{~nm}$ in Figure 1A is the same as that in Figure 1C, and it does not matter which procedure was used to get that ratio; the relative hues are invariant under multiplication by a constant.

We directly tested that assumption with a group of 6 subjects, each of whom scaled spectral lights with both $4+1$ and 5-category procedures in a counterbalanced design. In Figure $3 \mathrm{~A}$, the data points are the group's means for $4+1$ categories converted, as in Figure 1, to 5 categories; the curves are the means from direct use of the 5 -category procedures. The two procedures yield similar data when converted to the same form. We show in Figure 3B, for each subject, the differences between saturation values obtained from the two methods; the results are approximately the same for all individuals, and on average the differences are less than $5 \%$. Also, the differences do not change systematically across the spectrum. Note that the scale in Figure $3 \mathrm{~B}$ is magnified in comparison with $3 \mathrm{~A}$.

Either procedure is acceptable. And, in accord with subjects' preferences, we also found that the $4+1$ procedure was more reliable. For each subject, the mean of the variances for each category was always greater for the 5-category procedure.

\section{WITHIN-SUBJECTS RELIABILITY}

\section{Long-Term Stability}

Ideally, a psychophysical procedure should give results that remain stationary over time. Informally, we have looked at responses from many subjects and have found that they indeed remain stable. To examine this formally, we use data from a lengthy study of color vision across the retina in a group of 6 subjects, one of whom provided the results in Figure 1 (Abramov et al., 1991). The relevant data were from the 23 foveal stimuli that were included in the 115 experimental trials on each of 32 sessions spaced across several months. We averaged the results in blocks of four sessions, since foveal stimuli only appeared once per session. The symbols in Figures 4A and 4B show the group means for the first and last blocks, respectively; the curves are the means over all 32 sessions. The results from the beginning of the study agree closely with those at the end. To compare performance across blocks, we calculated the mean response for each category (e.g., R) for each block of four sessions; these means, which are proportional to the areas under their respective scaling curves, remain 
very stable across time (Figure $4 \mathrm{C}$ ). Although we show only group data, each individual showed the same degree of long-term stability. Although mean responses remained stable, there was a slight decrease in variance across sessions, suggesting that precision improves a little with extended practice.

\section{Context Effects}

If subjects are indeed giving "absolute" information about their sensations, then any stimulus should always be rated in the same way regardless of what preceded itprovided that duration of time between stimuli is long enough to allow any physiological effects, such as chromatic adaptation, to dissipate. However, it is well known that context can affect judgments; they may depend on the other stimuli presented in a session (e.g., Gescheider \& Hughson, 1991; Schneider \& Parker, 1990).

Our study allowed us to test the stability in time of hue and saturation scaling and also provided information about possible effects of the context in which stimuli are seen. Each session began with a series of "practice" or "warm-up" stimuli, all presented to the fovea; subsequent "experimental" trials included the same foveal stimuli, but they were randomly intermixed with peripheral stimuli of different sizes and eccentricities (Abramov et al., 1991). Thus, foveal trials from the experimental series could be preceded by stimuli that appeared far more desaturated, which might, in turn, have biased the ratings of the foveal stimuli.

In Figure 5 we show the differences between the mean ratings of foveal stimuli seen during practice and experimental trials. The data are the mean differences for the group, together with their standard errors. The differences are quite small (of the order of $5 \%$ ), but there is a systematic trend for the practice trials to appear slightly more saturated. When we examined the original data, we found that the hue responses were largely unaffected by this comparison; the differences are due to changes in apparent saturation. This robustness of hue categories agrees with subjects' reports about which scales are easier to use; many subjects report that judgments of saturation are more difficult to make, and they seem more likely to be influenced by context.

\section{Stimulus Range}

The range of stimuli used in a magnitude-estimation study can bias the derived psychophysical relationship (e.g., Marks, 1974; Poulton, 1979; Stevens, 1961). Does the spectral range of stimuli introduce a similar bias in our procedures? Using the same group of subjects as for Figure 3, we tested this in a fully counterbalanced design. In one session, subjects saw stimuli spaced in our usual 10-nm steps across the spectrum; in other sessions, stimuli were spaced every $5 \mathrm{~nm}$ and were confined to either short or long wavelengths in nonoverlapping ranges. The total number of stimuli in any session was approximately the same. In Figure 6, the symbols indicate the group means for the two restricted ranges, and the curves show the means from the full-range sessions. The differences are small and unsystematic, implying that hue scaling is unusually robust and unaffected by changes in stimulus range within a session.

These data also confirm the previous finding that context exerts little influence (Figure 4); in the study of Figure 5, the context could have biased the reported hues for example, in one session, all stimuli might have appeared as almost exclusively $R$ and $Y$. Finally, the smooth progression of data points in restricted-range sessions shows that the method is sensitive enough to permit clear distinctions among isolated stimuli differing only by $5 \mathrm{~nm}$.

\section{BETWEEN-SUBJECTS VARIABILITY}

It is a truism that no two people have exactly the same visual system. But, equally, all those who pass screening tests for normal color vision produce traditional psychophysical functions that are very similar. Is the same true for hue and saturation scaling functions? As with all psychophysical procedures, we come across subjects who, although motivated, do not perform well. In particular, a few subjects do not seem to use the numerical scales appropriately; they do not use continuous scales and simply describe stimuli as $100 \%$ of one or other of the hue categories, even after one or two practice sessions. We discard such subjects. Fortunately, we find them to be rare - well less than $5 \%$ of our potential subjects. Interesting variations among subjects could be due to real physiological differences, variations in experience with hue scaling or with other forms of sensory scaling, or individual cultural or linguistic experiences.

\section{Hue Scaling Experience}

We have compared the functions from a group of 6 highly experienced observers (the same subjects as for Figure 4) with functions from another group of subjects-ones who had not previously participated in any study of vision. Each of these "naive" subjects was simply given our standard instructions and generated a set of data (four repetitions per stimulus), all within a single 1-h session. The results are shown in Figure 7; the data points indicate the group averages from the naive subjects, and the curves indicate data from the experienced subjects. In order to separate possible differences between performance with hue categories and with apparent saturation, in Figure 7A we show the hue responses before rescaling by the associated values of saturation; in Figure 7B we show the saturation values. Except for the shortest wavelength, the agreement between the hue functions is good. There are, however, small but systematic differences in saturation from these particular groups. In short, experience seems to have a minimal influence on the group means generated by the subjects in our task. However, experience does matter in terms of variance. We averaged the variances of the individual subjects in the above groups within each sensory category; for each category, the mean of the indi- 
vidual variances of the naive subjects was approximately double that of the experienced subjects.

\section{Variability Among Experienced Subjects}

Averaging together data from a group may obscure important details. Our experience has been that there is relatively little variability among subjects in hue and saturation scaling - group averages nicely reflect behavior and serve to reduce noise. To illustrate the range of intersubject variability, we gathered results from 12 subjects who scaled a standard set of foveal stimuli, using our usual $4+1$ method; these stimuli were included in several different studies. We show the group means in Figure 8. To indicate population variation, the error boundaries are \pm 1 estimated standard deviation of the population. Further, the data have not been rescaled into a 5-category format, since we want to show the range of variation in the data in the form used by the subjects. However, each individual datum was modified by the arcsine transform, as described earlier. In all categories, the variation is quite small, with saturation showing the largest variation. This agrees with the subjects' reports about the relative difficulty of scaling it. We conclude that subjects use our percentage scales similarly and that there are no major idiosyncratic ways of assigning these numbers to sensations.

\section{Language}

Although there is considerable evidence that differences in language have little effect on the meanings of the unique hue terms, it is still possible that there may be subtle differences in their usage in our kind of hue scaling task. To explore such effects completely, we would have to use the hue terms of a subject's native language and conduct the entire experiment in that language. We focused on a more limited case: Are there linguistic biases when a bilingual subject, for whom English is the second language, uses English to scale sensations? In Figure 9, we compare results from 6 bilingual subjects (data points) with those from 6 monolingual English speakers (curves). The bilingual group included several different languages: Arabic, Chinese, Hebrew, Korean, and Urdu. In agreement with a similar comparison for Japanese speakers (Uchikawa \& Boynton, 1987), there are no large differences that can be ascribed to language background. Moreover, there are no systematic differences in between-subjects variance in either group.

\section{CONCLUSIONS}

In this paper we have shown that hue and saturation scaling can be used easily and reliably to describe color sensations. The method does not seem to be prone to many of the problems associated with magnitude estimation in other contexts. A possible reason is that we do not instruct subjects to consider previous sensations and the numbers they assigned to them when judging a particular stimulus. We simply ask them to compare sensa- tions across categories. Thus, subjects might not have assigned numbers within a category in strict ratios of sensations; but, provided they operated in the same way in each category, their statement of the relative hues allows a ratio scale of relative hue. Our method does not prevent absolute magnitude estimation within each category (Zwislocki \& Goodman, 1980). A peculiar virtue of hue scaling may be that it "taps," quite closely, central physiological mechanisms that are the bases for very distinct perceptual categories (Ratliff, 1976).

In one sense, the validity of our methods is obviouswe want to know what colors look like to our subjects. However, we must also go beyond face validity. In some of our previous work we have directly compared functions derived from hue scaling with functions from classical threshold or matching techniques. These include, for example, the Bezold-Brucke hue shift with intensity (Gordon \& Abramov, 1988), wavelength discrimination (Abramov, Gordon, \& Chan, 1990), and large-scale color differences (Chan, Abramov, \& Gordon, 1991). In those papers, we also noted that hue and saturation scaling not only provided appropriate functions, but did so with at least an order of magnitude savings of time, and it could be readily used in many circumstances.

The only method we have of knowing anything about the magnitude of the color sensation is by a process of mental estimation. Moreover, the fact that the observations can be carried out under normal conditions of viewing, using both eyes and without the interposition of any optical devices, adds enormously to the value of the results. (Wright, 1981, p.147)

\section{REFERENCES}

Abramov, I., \& Gordon, I. (1994). Color appearance: On seeing red -or yellow, or green, or blue. Annual Review of Psychology, 45 , 451-485.

abramov, I., Gordon, J., \& Chan, H. (1990). Using hue scaling to specify color appearance. Proceedings of the Society of Photo Optical Instrumentation Engineers, 1250, 40-51.

ABRAMOV, I., Gordon, J., \& ChAN, H. (1991). Color appearance in the peripheral retina: Effects of stimulus size. Journal of the Optical Society of America, A8, 404-414.

Abramov, I., Gordon, J., \& Chan, H. (1992). Color appearance across the retina: Effects of a white surround. Journal of the Optical Society of America, A9, 195-202.

BEARE, A.C. (1963). Color-name as a function of wavelength. American Journal of Psychology, 76, 248-256.

BERLIN, B., \& KAY, P. (1969). Basic color terms. Their universality and evolution. Berkeley, CA: University of California Press.

Boynton, R. M. (1979). Human color vision. New York: Holt, Rinehart \& Winston.

Boynton, R. M., \& Gordon, J. (1965). Bezold-Brucke hue shift measured by color-naming technique. Journal of the Optical Society of America, 55, 78-86.

Boynton, R. M., Schafer, W., \& Neun, M. A. (1964). Hue-wavelength relationship measured by color-naming method for three retinal locations, Science, 146, 666-668.

Chan, H., Abramov, I., \& Gordon, J. (1991). Large and small color differences: Predicting them from hue scaling. Proceedings of the Society of Photo Optical Instrumentation Engineers, 1453, 381-389.

De Valois, R. L., Abramov, I., \& JaCOBS, G. H. (1966). Analysis of response patterns of LGN cells. Journal of the Optical Society of America, 56, 966-977. 
EVANS, R. M. (1964). Variables of perceived color. Journal of the Optical Society of America, 54, 1467-1474.

Fuld, K., Werner, J. S., \& Wooten, B. R. (1983). The possible elemental nature of brown. Vision Research, 23, 631-637.

Fuld, K., Wooten, B. R., \& Whalen, J. J. (1981). The elemental hues of short-wave and extraspectral lights. Perception \& Psychophysics, 29, 317-322.

Gescheider, G. A. (1988). Psychophysical scaling. Annual Review of Psychology, 39, 169-200.

Gescheider, G. A., \& Bolanowski, S. J., JR. (1991). Final comments on ratio scaling of psychological magnitudes. In S. J. Bolanowski, Jr. \& G. A. Gescheider (Eds.), Ratio Scaling of Psychological Magnitude (pp. 295-311). Hillsdale, NJ: Erlbaum.

Gescheider, G. A., \& Hughson, B. A. (1991). Stimulus context and absolute magnitude estimation: A study of individual differences. Perception \& Psychophysics, 50, 45-57.

Gordon, J., \& ABRAMOV, I. (1977). Color vision in the peripheral retina: II. Hue and saturation. Journal of the Optical Society of America, 67, 202-207.

GoRDON, J., \& ABRAMOv, I. (1988). Scaling procedures for specifying color appearance. Color Research \& Application, 13, 146-152.

Graham, C. H. (1958). Sensation and perception in an objective psychology. Psychological Review, 65, 65-76.

HARD, A., \& Sivik, L. (1981). NCS-Natural Color System: A Swedish standard for color notation. Color Research \& Application, 6, $129-138$.

HEIDER, E. R. (1972). Universals in color naming and memory. Journal of Experimental Psychology, 93, 10-20.

HeRING, E. (1920). Grundzuge der Lehre vom Lichtsinn [Outlines of a theory of the light sense]. Berlin: Julius Springer.

HunT, R. W. G. (1975). The reproduction of colour. Kings Langley, England: Fountain Press.

Hurvich, L. M. (1981). Color vision. Sunderland, MA: Sinauer Associates.

JACOBS, G. H., \& GAYLORD, H. A. (1967). Effects of chromatic adaptation on color naming. Vision Research, 7, 645-653.

Jameson, D., \& Hurvich, L. M. (1959). Perceived color and its dependence on focal surrounding, and preceding stimulus variables. Journal of the Optical Society of America, 49, 890-898.

KAISER, P. K. (1968). Color names of very small fields varying in duration and luminance. Journal of the Optical Society of America, 58, 849-852.

KaY, P., \& MCDANIEL, C. K. (1978). The linguistic significance of the meanings of basic color terms. Language, 54, 610-645.

LURIA, S. M. (1967). Color-name as a function of stimulus-intensity and duration. American Journal of Psychology, 80, 14-27.

Marks, L. E. (1974). Sensory processes. New York: Academic Press.

Munsell, A. H. (1905). A color notation. Boston: Ellis.

Newhall, S. M., Nickerson, D., \& Judd, D. B. (1943). Final report of the O.S.A. subcommittee on the spacing of the Munsell colors. Journal of the Optical Society of America, 33, 385-418.

NEWTON, I. (1704). Opticks: Or a treatise of the reflexions, refrac- tions, inflexions and colours of light. London: Sam. Smith and Benj. Walford.

PoKorny, J., \& SMITH, V. C. (1977). Evaluation of single-pigment shift model of anomalous trichromacy. Journal of the Optical Society of America, 67, 1196-1209.

Poulton, E. C. (1979). Models for biases in judging sensory magnitude. Psychological Bulletin, 86, 777-803.

QuinN, P. C., Rosano, J. L. \& Wooten, B. R. (1988). Evidence that brown is not an elemental color. Perception \& Psychophysics, 43, 156-164.

RatLIFF, F. (1976). On the psychophysiological bases of universal color terms. Proceedings of the American Philosophical Society, 120, 311-330.

Schneider, B., \& Parker, S. (1990). Does stimulus context affect loudness or only loudness judgments? Perception \& Psychophysics, 48, 409-418.

Smith, V. C., Pokorny, J., \& Swartley, R. (1973). Continuous hue estimation of brief flashes by deuteranomalous observers. American Journal of Psychology, 86, 115-131.

Sobagaki, H., Yamanaka, T., Takahama, K., \& Nayatani, Y. (1974). Chromatic-adaptation study by subjective-estimation method. Journal of the Optical Society of America, 64, 743-749.

Sternheim, C. E., \& Boynton, R. M. (1966). Uniqueness of perceived hues investigated with a continuous judgmental technique. Journal of Experimental Psychology, 72, 770-776.

STEVENS, S. S. (1961). The psychophysics of sensory function. In W. A. Rosenblith (Ed.), Sensory communication (pp. 1-33). New York: Wiley.

TelLeR, D. Y. (1990). The domain of visual science. In L. Spillmann \& J. S. Werner (Eds.), Visual perception: The neurophysiological foundations (pp. 11-21). San Diego, CA: Academic Press.

UChIKAWA, K., \& BOynton, R. M. (1987), Categorical color perception of Japanese observers: Comparison with that of Americans. Vision Research, 27, 1825-1833.

WINER, B. J. (1971). Statistical principles in experimental design. New York: McGraw-Hill.

WRIGHT, W. D. (1981). Why and how chromatic adaptation has been studied. Color Research \& Application, 6, 147-152.

WyszeCKI, G., \& StIles, W. S. (1967). Color science. New York: Wiley.

Zrenner, E., Abramov, I., Akita, M., Cowey, A., Livingstone, M., \& VALBERG, A. (1990). Color perception: Retina to cortex. In L. Spillmann \& J. S. Werner (Eds.), Visual perception: The neurophysiological foundations (pp. 163-204). San Diego, CA: Academic Press.

ZwisLOCKI, J. J. (1983). Group and individual relations between sensation magnitudes and their numerical estimates. Perception \& Psychophysics, 33, 460-468.

ZwISLOCKI, J. J., \& GoODMAN, D. A. (1980). Absolute scaling of sensory magnitudes: A validation. Perception \& Psychophysics, 28, 28-38.

(Manuscript received April 7, 1993; revision accepted for publication October 25,1993 .) 